\title{
Observando las alianzas de colaboración entre Organizaciones Sin Fines de Lucro y Organizaciones Estatales Chilenas ${ }^{1}$
}

\author{
Carolina Muñoz Guzmán \\ Pontificia Universidad Católica de Chile, Santiago, Chile. Email: cmunozgu@uc.cl
}

\section{Paula Miranda Sánchez}

Pontificia Universidad Católica de Chile, Santiago, Chile. Email: paulamiranda@uc.cl

\section{Rodrigo Flores Guerrero}

Pontificia Universidad Católica de Chile, Santiago, Chile. Email: rfloresu@uc.cl

Resumen: Situados en un progresivo traspaso de responsabilidades en la ejecución de los servicios sociales desde el Estado hacia actores no gubernamentales, aparece una demanda hacia el mismo Estado, el cual en lugar de simplemente declinar su labor, debiera de hecho expandir su regulación de modo de asegurar que la oferta social sea eficiente en satisfacer al ciudadano usuario en aquellos servicios prometidos por las políticas y programas sociales. Así mismo, la institucionalidad pública debiera reconocer las demandas del mundo privado para actuar articuladamente hacia fines comunes, que finalmente conduzcan a un horizonte de desarrollo de una sociedad más igualitaria. Este estudio da cuenta de las tensiones, reveladas por autoridades de servicios públicos que atienden personas vulnerables, en su relación de colaboración con OSFL (Organizaciones Sin Fines de Lucro), para alcanzar tal horizonte.

Palabras clave: OSFL, Estado, colaboración

\section{Observing partnership between nonprofit organizations and State organizations}

\begin{abstract}
The progressive transfer of responsibilities in social service implementation from state to nongovernmental actors, brings a demand to the same state, who instead of reducing its labor, should develop adequate mechanisms of regulation to ensure efficiency in social offer, in order to satisfy citizens demands in those public political commitments. Likewise, public institutions should recognize the demands from the private world to act articulated towards common goals, which ultimately would lead to a horizon of development of a more egalitarian society. This study reports on the tensions revealed by social services authorities serving vulnerable people, in regards to their collaborative relationship with Non Profit Organizations, in order to reach that horizon.
\end{abstract}

Key words: Nonprofit organizations, State, collaboration 


\title{
Observando a parceria entre as organizações sem fins lucrativos e organismos do Estado
}

\begin{abstract}
Resumo: A progressiva transferência de responsabilidades na implementação do serviço social do Estado para atores não-governamentais, traz uma demanda para o mesmo estado, que em vez de reduzir o seu trabalho, devem desenvolver mecanismos adequados de regulação para garantir a eficiência na oferta social, a fim de satisfazer as demandas dos cidadãos desses compromissos públicos políticos. Da mesma forma, as instituições públicas devem reconhecer as demandas do mundo particular para agir articulado com metas comuns, que em última análise, levariam a um horizonte de desenvolvimento de uma sociedade mais igualitária. Este estudo relata as tensões revelada por autoridades dos serviços sociais que atendem pessoas vulneráveis, no que diz respeito à sua relação de colaboração com organizações sem fins lucrativos, para chegar a esse horizonte.
\end{abstract} tado.

Palavras-chave: organizações sem fins lucrativos, a colaboração do Es-

$$
* * *
$$

\section{Introducción}

La transformación de los servicios sociales estatales en Chile desde el retorno a la democracia ha Estado demarcada por un énfasis en la perspectiva de derechos. Los principios garantistas y universalistas, privilegiados en este enfoque, conducentes hacia la constitución de ciudadanía, deben imbricarse en la oferta de sistemas de servicios complejos, situación que hoy no parecieran sintonizar con una regulada relación entre Estado y las OSFL que ejecutan las tradicionales tareas que antaño realizaba el Estado.

Situados en un progresivo traspaso de responsabilidades en la ejecución de los servicios sociales desde el Estado hacia actores no gubernamentales, aparece una demanda hacia el mismo Estado, el cual en lugar de simplemente declinar su labor, debiera de hecho expandir su regulación de modo de asegurar que la oferta social sea eficiente en satisfacer al ciudadano usuario en aquellos servicios prometidos por las políticas y programas sociales. Así mismo, la institucionalidad pública debiera reconocer las demandas del mundo privado para actuar articuladamente hacia fines comunes, que finalmente condujeran a un horizonte de desarrollo de una sociedad más igualitaria.

Se requiere entonces de una oferta que responda adecuadamente a la demanda social, en el escenario de una sociedad democrática de mercado donde las personas demandan transparencia de los servicios estatales así como mayor eficiencia, como una forma de expresión de sus derechos ciudadanos (Wormald \& Brieba 2010), el logro de este horizonte requiere necesariamente la entrega servicios públicos coordinados en torno a las necesidades de las personas, es decir se trata de servicios integrados, menos duplicados, con menos retraso y más eficientes. 
Este estudio exploratorio indaga preliminarmente sobre los ejes de colaboración y complejidad de las organizaciones, establecidas por las OSFL con los establecimientos del Estado bajo el supuesto de que esta relación está condicionada por características institucionales y de los actores que participan en la relación de colaboración, características que tenderían a entorpecer la gestión pública. Con el objetivo de estudiar esta premisa se desarrolló un panel de expertos, constituido por autoridades de cinco servicios públicos encargados de ejecutar programas sociales, para lo cual establecen alianzas con y OSFL's, que implementan intervenciones sociales con personas en situación de vulnerabilidad social. Esta constituye la primera etapa de un estudio que se profundizará en sucesivas etapas de investigación.

Los servicios sociales convocados fueron Servicio Nacional del Adulto Mayor (SENAMA), Departamento de Protección del Servicio Nacional de Menores (DEPRODE), Servicio de Vivienda y Urbanismo (SERVIU) y Servicio Nacional de la Mujer (SERNAM) y el Fondo Nacional de Inversión Social (FOSIS); la necesidad de estudiar esta relación se sustenta en el ya identificado desafío de la coordinación de la política social y el reconocimiento de que en la estructura vigente hoy día hay debilidad respecto de las atribuciones y capacidades reales para asegurar la eficiencia y eficacia de la política social (Centro de Políticas Públicas UC, 2011).

En el caso de las relaciones del Estado con las OSFL entendemos, desde la perspectiva sistémica, a las organizaciones públicas como parte del aparato estatal o dependientes de él, encargándose de dar solución de los problemas que les han sido delegados por este aparato. Dado que los problemas de la sociedad son complejos, difusos y no admiten especificaciones (Rodríguez, 2002), las organizaciones públicas dejan parte importante de dichos problemas sin resolución. Esta es la razón por la cual emerge un tercer sector, constituido por organizaciones no gubernamentales que buscan enfrentar los problemas insuficientemente tratados por las organizaciones públicas gubernamentales, y OSFL's, que velan por problemas dejados de lado por las organizaciones privadas con fines de lucro. Independientemente de esta diferenciación, todas ellas se caracterizan por no ser dependientes del Estado ni perseguir fines de lucro y por eso se las puede denominar indistintamente organizaciones no gubernamentales $\mathrm{u}$ OSFL. Nos interesa en esta etapa de la investigación identificar las principales dimensiones que tensionan la relación de colaboración entre estas organizaciones.

\section{Pilares de la colaboración inter-organizacional}

La piedra angular para modernizar los servicios sociales se ha descrito como la asociación y la colaboración (Glendinning 2000; Robinson \& Cottrel 2005), éstas aparecen como mecanismos básicos para lograr traba- 
jo conjunto orientado hacia metas comunes específicas. Gazley y Brudney (2007) definen la colaboración inter-organizacional como un proceso por el cual las organizaciones que atienden un problema común, buscan conjuntamente soluciones que no pueden alcanzarse trabajando asiladamente. La colaboración requiere una participación voluntaria, autónoma (los participantes mantienen su independencia en la toma de decisiones, aunque acuerden algunas reglas comunes); y tiene un propósito transformador. Esta definición excluye relaciones que son puramente contractuales, donde el poder no es compartido, y donde existe una asociación que no comparte metas específicas.

Gannon-Leary, Baines y Wilson (2006) han señalado que las dimensiones de visión y compromiso resultan fundamentales en el sentido de que las organizaciones tengan clara la misión de la cooperación y las metas realizables que se desprenden de ésta. De allí que como lo han establecido Licha y Molina (2006) la coordinación no es un fin en sí misma, sino un instrumento estratégico que apunta hacia los objetivos de la política social, favoreciendo el logro de fines centrales de la gerencia social, afectando "no sólo el qué y cómo sino también el por qué y para qué de la gerencia social” (Ibid:iii). En este sentido Peters (1998) establece una responsabilidad central en el rol del Estado para liderar las políticas desde un conjunto común de valores e ideas en el marco de una 'integración de políticas' que supere la pura oferta de servicios para alcanzar un nivel de cooperación inter-organizacional que haga compatibles las misiones de las partes y que se imponga a las contradicciones que impidan contar con un imaginario común sobre el horizonte al que se aspira.

En vista de lo anterior, la centralidad de los objetivos de la política social y de la gerencia social requieren del compromiso y la comprensión común en todos los niveles de las organizaciones involucradas en la colaboración, no sólo en las cúpulas organizacionales; en este sentido daría lo mismo desarrollar estrategias comunes si no hay un cambio significativo en los trabajadores de primera línea, o implementadores (Banks 2002: 9), lo cual supone evidenciar el valor público de los eslabones que articulan la acción pública como 'una cadena virtuosa que establece relaciones causales entre las tareas cotidianas de las organizaciones involucradas y las finalidades últimas de la acción gubernamental’ (Repetto 2005:32).

Hacer posible la concordancia entre las organizaciones en todos sus niveles, de modo que la colaboración pueda ser efectiva, supone una reflexión en torno al liderazgo o estilo de gobierno que guía la alianza colaborativa. Para el desarrollo de las estructuras de colaboración interagencial es necesario contar con apropiadas estructuras de gobierno (Huxham \& Vangen 2000; Sullivan \& Skelcher 2002) que faciliten la resolución de conflictos generados entre las agencias en torno al ejercicio de roles, sus límites y tensiones que emerjan debido a distintos protocolos de acción, valores, temor a que la colaboración afecte el poder, estatus y seguridad de cada agencia. Así mismo afectan las diferencias en los criterios de elegibilidad, los roles profesionales desempeñados en cada agencia, la dis- 
tribución del poder y los niveles de participación de cada agencia. Según Peters (1998) la estrategia que logra minimizar los riesgos señalados pone en el centro el liderazgo y medios de coordinación bien formalizados; el autor se refiere a líderes especialistas capaces de dar una marco y re-enmarcar cada vez que sea necesario los temas que amplían la capacidad de las partes para colaborar y producir soluciones estratégicas a los problemas, es decir que una colaboración significativa sólo puede ser lograda a través del desarrollo de un marco de referencia común entre los participantes del proceso, pero no cualquier marco de referencia, si no uno que convenza a los participantes que ellos ganan algo, o al menos, no están perdiendo con esta colaboración (Peters, Ibid). Como lo han planteado Scott and Thurston (2004) la explicitación de la forma en que se ordenan las relaciones de autoridad, cómo se toman las decisiones, los canales de participación y la forma de planificar y distribuir los recursos es un tipo de formalización fundamental. En estos ámbitos, las autoras indican que las instituciones donde priman relaciones burocráticas y patriarcales no permiten generalmente un trabajo relacional con las otras partes, a pesar de que los agentes de las instituciones tengan la voluntad de trabajar colaborativamente, el peso de la estructura obstaculiza la colaboración.

Sumado a las dimensiones ya expuestas, vale la pena agregar un punto relativo a las personas y la intervención. La colaboración depende de la manera en que las presiones son resueltas en el trabajo, así es necesario que los directivos manejen adecuadas relaciones inter-organizacionales e interpersonales, que sean favorables al intercambio. Sin embargo, los servicios se enfrentan a agendas sobrecargadas y cuentan con poco espacio para trabajo conjunto, una vez que se han establecido las políticas, procesos y estructuras de la colaboración inter-agencial, no es automático que profesionales que históricamente han estado separados trabajen coordinadamente. Compartir estrategias de trabajo requiere superar perspectivas estrechas del profesionalismo y del particularismo de cada organización. Para esto, identificar un individuo con habilidades para actuar como enlace entre las personas de los distintos sectores ayuda al proceso de asociación (Mattessich \& Monsey 1992; Mullett et al. 2002). El mayor desafío es involucrar a los trabajadores de primera línea, quienes tienden a evaluar el trabajo inter-agencial simplemente como más trabajo; como se ha sostenido anteriormente aquí el trabajo de los líderes de los equipos es fundamental.

De lo anterior se deriva que un foco de atención para la colaboración es la congruencia de las culturas organizacionales y el logro de un progresivo consenso entre las partes respecto del significado, el propósito, los principios bajo los que opera la asociación, y los criterios que determinarán el éxito (Scott \& Thurston 2004). Esto último supone tener una visión compartida sobre el sujeto de atención, para lo cual reflexionar y discutir es parte de la tarea de colaboración. En este sentido el tema del tiempo de la relación colaborativa no deja de ser relevante, la tarea de colaboración consume mucho tiempo, por lo tanto deben existir proyectos de lar- 
go plazo que permitan fundar ventajas colaborativas atractivas para los participantes, se requieren desarrollar adecuados patrones de comunicación, establecer y asumir responsabilidades y acordar una visión común sobre los usuarios.

\section{Problema de investigación y metodología}

El propósito de este estudio es identificar las principales dimensiones que favorecen y/o tensionan la relación de colaboración entre las organizaciones del Estado y las OSFL desde una perspectiva organizacional. Para ello la pregunta central planteada en el Panel de expertos fue ¿cuáles son los principales obstáculos y facilitadores que ustedes identifican en la relación de colaboración que mantienen con las OSFL que implementan los servicios que sus organizaciones mandatan?

Las sub-preguntas guiando esta exploración fueron:

1. ¿Cuáles son, fuera de los Términos de Referencia ${ }^{2}$, las exigencias, atributos, características que se solicitan a los colaboradores?

2. ¿Constituyen las prácticas de intervención de los colaboradores un insumo para la petición de colaboración?

3. ¿Cómo se resuelven los asuntos relativos a recursos?

4. ¿Cómo se evalúa la relación de colaboración?

Estas preguntas fueron discutidas en un panel de expertos constituido por autoridades de servicios sociales ya identificados, con el objeto de evaluar el grado de acuerdo o disenso en relación al principal tema discutido y las sub-preguntas. Los participantes contribuyeron con sus visiones sobre cada tema discutido, los facilitadores del panel agruparon los principales temas que emergieron de la discusión y estos fueron nuevamente discutidos por el grupo para clarificar y evaluar cada idea.

El análisis de la información recogida constituyó un corpus al cual se le aplicó análisis temático, con el objeto de identificar, analizar y reportar patrones (temas) dentro del corpus. El análisis temático permite la organización y descripción de la información en forma detallada y además facilita la interpretación de variados aspectos considerados en la investigación (Boyatzis 1998). El procedimiento usado siguió el sugerido por esta técnica de análisis: la información del panel de expertos fue grabada y transcrita con precisión, el corpus obtenido se codificó asignando relevancia similar a cada ítem de datos, se evitó considerar temas que emergieran de anécdotas, sino más bien se desarrolló un proceso de codificación inclusivo y comprensivo. Los temas fueron chequeados entre ellos, buscando consistencia interna facilitando una interpretación analítica que pudiera ser ilustrada por extractos, y se desarrolló un reporte escrito que permitió la construcción de este artículo (Braun \& Clarke 2006). 


\section{Descripción y análisis de la información}

Los temas centrales que emergieron del panel de expertos tendieron más bien hacia la homogeneidad, con ciertas diferencias que estuvieron determinadas por las funciones desarrolladas por los servicios y el tipo de prestación que otorgan. Los extractos usados para el análisis se encuentran en el Anexo 1.

En primer lugar la relación de cada servicio estudiado con las OSFL que implementan las prestaciones de servicios estuvieron mediadas por un recurso jurídico, el que norma, limita y determina el tipo de organización y de relación que cada servicio público establece con las OSFL. El recurso jurídico busca diferentes objetivos en cada servicio y su relevancia tiene diferentes grados de impacto. En el caso de las instituciones más antiguas como el SERVIU y el FOSIS surge la necesidad de una regulación cada vez más estricta desde el Estado, dado que se relacionan con instituciones que se van transformando en contrapartes que traen sus propios incentivos, no sólo alejados de la misión y visión del servicio que representa sino también del tipo de ejecución esperable. En el caso de las instituciones más nuevas como SENAMA y SERNAM, el recurso jurídico tiende a velar por los principios orientadores del servicio, que sean compartidos pero la regulación aparece más laxa. Esto se evidencia en los propósitos, por ejemplo, de SENAMA de iniciar un proceso más regulado, desde la Dirección de Presupuesto $^{3}$, en el establecimiento de criterios de funcionamiento que habiliten la permanencia de una organización colaboradora como tal.

El tema de la especialización aparece sólo en algunos discursos (SERVIU, DEPRODE, FOSIS) como un requisito fundamental para el Estado y a la vez como problema que acarrea la centralización del país, es decir existe escasa oferta especializada en regiones. Este tema además tiende a entrar en tensión con la sobrevivencia de las organizaciones, que en algunos casos se tornan en 'multipropósito' para asegurar financiamiento. El financiamiento juega un doble rol, ya que permite la permanencia de las organizaciones en el sistema asegurando ciertos niveles de especialización (como es el caso de SERVIU), pero a la vez debilita esa misma especialización cuando las organizaciones quieren diversificar sus servicios, para asegurar ingresos (FOSIS, DEPRODE).

La heterogeneidad de las OSFL que implementan programas juega un rol en servicios sociales como DEPRODE, limitando espacios para la innovación y poniendo cuestionamientos en el servicio sobre los grados de autonomía de las contrapartes. El énfasis en una política nacional pareciera entrar en tensión con las especificidades (riquezas y debilidades) locales y la incertidumbre de poder delegar mayor autonomía en los centros regionales. En este sentido existe una cierta desconfianza en las capacidades locales para emprender con independencia algunos modelos de intervención, que se conjuga con una valoración por el saber acumulado de algunas organizaciones, en una relación tensionada. Los mecanismos de evaluación parecen no siempre favorecer la resolución de esta tensión ya que la oferta de 
servicios no sólo depende de la oferta local que, como se ha dicho, es heterogénea.

Respecto a la relación del Estado con la OSFL como una organización que contiene diversos niveles, centrales, de diseño y ejecutivos, no aparece en el discurso de ningún servicio una preocupación por asegurar compromiso y la comprensión común en todos los niveles de las organizaciones involucradas en la colaboración, más bien pareciera existir una relación con las cúpulas organizacionales, lo cual, siguiendo a Banks (2002), acarrea consecuencias negativas en el alineamiento con estrategias comunes en los trabajadores de primera línea, limitando el éxito de una cadena virtuosa en las acciones cotidianas de la acción pública. Esta situación se imbrica con las responsabilidades declaradas por los servicios respecto a monitorear, supervisar y entregar asistencia técnica a sus contrapartes, ya que la existencia de medios de coordinación bien formalizados -a excepción del SERVIU- aparece sólo declarada pero no precisada por los expertos, situación que también se relaciona a los niveles de centralismo del país, donde los poderes representativos y de toma de decisiones son muy restringidos en los niveles locales. Aparece aquí una señal de la primacía de instituciones con relaciones burocráticas que entorpecen un trabajo relacional con las otras partes, no obstante los agentes de las instituciones tengan la voluntad de trabajar colaborativamente, el peso de la estructura obstaculiza la colaboración (Scott and Thurston 2004).

La situación señalada se evidencia cuando por ejemplo en SERNAM se declara la dificultad de implementar las estrategias desde la visión de la política pública, debido al peso que impone la misión institucional, la superación de esta tensión pasa por formalizar medios de coordinación y asegurar liderazgos que comprometan a los ejecutores con la visión del Estado. Este requerimiento se conjuga a la vez, en el discurso de los expertos, con la necesidad de escuchar el 'feedback' de las contrapartes, en este sentido por ejemplo en DEPRODE las relaciones más horizontales con los organismos colaboradores está mediado por sus niveles de validación que el servicio público otorga a la contraparte al reconocer que es un buen gestor, que tiene trayectoria, que ha logrado innovar en un área específica, o porque es experto en algunos temas, en función de un método o de un modelo de intervención concreto, se reconoce aquí un tipo de relación (horizontal en lugar de vertical) que favorece el funcionamiento y operatividad de los proyectos.

Como se señaló antes, el financiamiento afecta la permanencia de las organizaciones en la prestación de un mismo servicio, y este último tópico determina de sobremanera la relación de colaboración, no sólo en apuntar hacia una misión común, sino también en conceptualizar el sujeto de intervención desde una perspectiva similar y compatible. Se produce una distancia, no exenta de cegueras, entre los la política social, los Términos de Referencia y la implementación, por parte del tercero mandatado. 


\section{Conclusiones finales}

Las principales tensiones identificadas en el relato de los expertos del sector público respecto a su relación de colaboración con las OSFL son las siguientes:

a. Misión institucional v/s Misión de la Política Pública, el logro de los objetivos de la política social dependen de una sintonía entre misiones y visiones institucionales: Siguiendo a Peters (1998) existiría aquí una deficiencia en el rol de liderazgo desde el Estado que asegure un marco de integración en base a valores e ideas comunes que determine la dirección de la colaboración.

b. Flexibilidad v/s Inflexibilidad de la normativa que rige la relación de colaboración, que por un lado asegura el cumplimiento de regulaciones fundamentales del accionar público, especialmente en FOSIS y MINVU, pero que a veces deja ejecutores de excelencia fuera de las licitaciones. O como en el DEPRODE, mejora la competencia en base términos de referencia, sin embargo los buenos ejecutores no siempre saben formular proyectos, ya que a veces esto último se transforma en un nuevo "giro del negocio".

c. Heterogeneidad en la calidad de los prestadores, reduce las posibilidades de establecer relaciones horizontales de colaboración y asignar niveles de autonomía en la gestión de políticas.

d. Medios de coordinación bien formalizados v/s simples declaraciones del acuerdo de colaboración; la relación de asesoría técnica, de monitoreo y seguimiento se pierde en la formalidad del contrato de colaboración, pero no logra estamparse en procedimientos operativos que permitan el ampliar la capacidad de las partes para colaborar y producir soluciones estratégicas a los problemas que van enfrentando.

e. Centralización y calidad de los ejecutores. Se producen no sólo heterogeneidades en la calidad de los servicios sino también la oferta de algunos servicios se limita en algunas regiones porque no hay prestadores especializados.

f. Financiamiento v/s permanencia y calidad de las prestaciones, la lógica de la licitación privada ha producido un nuevo estilo de vínculo con las OSFL que se especializan en el diseño de proyectos para asegurar financiamiento, y de este modo la permanencia de ejecutores de calidad no siempre es fortalecida, por el contrario la tendencia pareciera ser que se pierden buenos ejecutores porque la licitación privilegia el buen diseño, que frente a un monitoreo débil parece predominar a la hora de seleccionar a los colaboradores.

La necesidad de sobrevivencia de las organizaciones, su tendencia al multipropósito, la fragilidad de los procesos de seguimiento, monitoreo 
y asesoramiento técnico desde el Estado, sumado a debilidades en la especialización de las organizaciones, producto de las mismas circunstancias descritas antes, tornan la relación en un círculo de afectación mutua y que requiere ser atendido con urgencia para que la relación de colaboración Estado-OSFL sea fructífera en términos de alcanzar los objetivos dispuestos por la política pública. Las tendencias y tensiones identificadas en los servicios estudiados aquí permitirán orientar las futuras etapas de investigación del estudio Observando la colaboración inter-organizacional: El caso de las alianzas de colaboración entre Organizaciones Sin Fines de Lucro y Organizaciones Estatales en Chile. 


\section{Notas}

${ }^{1}$ Este estudio corresponde a la primera etapa del Proyecto Fondecyt $\mathrm{N}^{\circ} \mathrm{N}^{\circ} 1110416$ cuyo investigador principal es Rodrigo Flores, y es parte de las investigaciones presentadas en la VII Conferencia Regional de ISTR para América Latina y el Caribe, realizada en Julio1214-2011.

${ }^{2}$ Entendidos como especificaciones técnicas, lineamientos, objetivos de cómo ejecutar un determinado trabajo, mandatado.

${ }^{3}$ Organismo dependiente del Ministerio de Hacienda cuya misión es velar por la eficiente asignación y uso de los recursos públicos en el marco de la política fiscal, mediante la aplicación de sistemas e instrumentos de gestión financiera, programación y control de gestión. Así, es responsable de formular la Ley de Presupuestos, realizar modificaciones al presupuesto vigente por medio de decretos, elaborar los programas de caja mensuales y registrar el gasto mensual, efectivo y devengado por Institución. 


\section{Bibliografía}

Boyatzis, R. E. (1998), Transforming qualitative information: Thematic analysis and code development. Thousand Oaks, CA: Sage.

Banks, P. (2002), Partnerships under pressure, King's Fund, London

Braun, Virginia and Clarke, Victoria (2006), Using thematic analysis in psychology. Qualitative Research in Psychology, 3 (2). pp. 77-101”. Qualitative Research in Psychology is available online at: http:// www.informaworld.com/smpp/content ${ }^{\sim} \mathrm{db}=\mathrm{all}^{\sim}$ content $=\mathrm{a} 795127197^{\sim}$ frm=titlelink

Centro de Políticas Públicas UC (2011), “Nueva institucionalidad social: continuidad y cambios”, Enero 2011 n 7 Análisis del proyecto de ley que crea el Ministerio de Desarrollo Social (Boletín 7196- 06) Documento. PUCCH Santiago de Chile

Gazley, B. and Brudney, J. (2007), The Purpose (and Perils) of GovernmentNonprofit Partnership Nonprofit and Voluntary Sector Quarterly2007; 36; 389

Gannon-Leary, P.; Baines, S.; Wilson, R. (2006), Collaboration and partnership: A review and reflections on a national project to join up local services in England, J Interprof Care. 2006 Dec; 20(6):665-74.

Glendinning, C. (2000), "Partnership between Health and Social Services: Developing a framework for evaluation”. Policy and Politics 30 , pp. 115127

Huxham, C. and Vangen, S. (2000), Leadership in the shaping and implementation of collaboration agendas: How things happen in a (not quite) joined-up world. Academy of Management Journal 43 , pp. 11591176

Licha, Isabel y Molina, Carlos (2006), Coordinación de la Política Social: criterios para avanzar, Serie de Documentos de Trabajo I-58 Documentos de trabajo del INDES, Washington, D.C.

Mattessich, P. W. and Monsey, B. R. (1992), Collaboration: What makes it work? Amherst Wilder Foundation, St. Paul, MN

Mullett, J., Jung, K. and Hills, M. (2002), “Collaboration in non-profits: Dynamics and dilemmas”. Canadian Review of Social Policy 49 - 50 , pp. 155-173.

Peters, Guy (1998), The Search for Coordination and Coherence in Public Policy: Return to the Center? Department of Political Science University of Pittsburgh http://userpage.fu-berlin.de/ffu/akumwelt/bc2004/download/ peters_f.pdf 
Reppetto, Fabián (2005), “Introducción: La gerencia social y el complejo escenario de la política social en América Latina”. En La gerencia social ante los nuevos retos del desarrollo social en América Latina. INDES; INAP, BID Norwegian Ministry of Foreign Affairs, Guatemala 2005.

Robinson, M. and Cottrel, D. (2005), "Health professionals in multidisciplinary and multi-agency teams: Changing professional practice”. Journal of Interprofessional Care 19 , pp. 547-560.

Rodríguez, Darío (2002), Gestión Organizacional. Elementos para su Estudio. Ediciones Universidad Católica de Chile, Santiago-Chile

Scott, C; Thurston W (2004), “The Influence of Social Context in Partnerships on Canadian Health Systems Gender”, Work \& Organization Volume 11, Issue 5, pages 481-505, September 2004

Sullivan, H. and Skelcher, C. (2002) Working across boundaries Macmillan Palgrave, Basingstoke

Wormald \& Brieba, (2010) Institutional change and development in Chilean market society http://cmd.princeton.edu/papers/wp1003c.pdf 